\title{
The NtrY/NtrX System of Sinorhizobium meliloti GR4 Regulates Motility, EPS I Production, and Nitrogen Metabolism but Is Dispensable for Symbiotic Nitrogen Fixation
}

\author{
Nieves Calatrava-Morales, Joaquina Nogales, Kinia Ameztoy, Bart van Steenbergen, and María J. Soto \\ Department of Soil Microbiology and Symbiotic Systems, Estación Experimental del Zaidín, Consejo Superior de \\ Investigaciones Científicas (CSIC), Profesor Albareda 1, 18008 Granada, Spain
}

Accepted 8 April 2017.

\begin{abstract}
Sinorhizobium meliloti can translocate over surfaces. However, little is known about the regulatory mechanisms that control this trait and its relevance for establishing symbiosis with alfalfa plants. To gain insights into this field, we isolated Tn5 mutants of S. meliloti GR4 with impaired surface motility. In mutant strain GRS577, the transposon interrupted the $n t r Y$ gene encoding the sensor kinase of the NtrY/NtrX two-component regulatory system. GRS577 is impaired in flagella synthesis and overproduces succinoglycan, which is responsible for increased biofilm formation. The mutant also shows altered cell morphology and higher susceptibility to salt stress. GRS577 induces nitrogenfixing nodules in alfalfa but exhibits decreased competitive nodulation. Complementation experiments indicate that both $n t r Y$ and $n t r X$ account for all the phenotypes displayed by the $n t r Y:$ Tn 5 mutant. Ectopic overexpression of VisNR, the motility master regulator, was sufficient to rescue motility and competitive nodulation of the transposant. A transcriptome profiling of GRS577 confirmed differential expression of exo and flagellar genes, and led to the demonstration that NtrY/NtrX allows for optimal expression of denitrification and nifA genes under microoxic conditions in response to nitrogen compounds. This study extends our knowledge of the complex role played by NtrY/NtrX in S. meliloti.
\end{abstract}

Motility is a feature of great importance in bacterial ecology. The ability to move provides bacteria with an increased efficiency to acquire nutrients and avoid toxic substances, as well as the capacity to approach and colonize potential hosts. However, the costs of motility are significant due to the metabolic burden of synthesizing flagellar components and the energetic expense of fueling flagellar motors, but also as an alerting signal of the host immune system. Therefore, it is not surprising that both the synthesis and function of flagella are subject to strict control.

Rhizobia are motile bacteria that can exist either as free-living organisms in the soil or in symbiotic association with their host legume plants, in which they induce the formation of nitrogen-

Corresponding author: M. J. Soto; Telephone: +34-958181600 ext. 150; Fax: +34-958181609; E-mail: mariajose.soto@eez.csic.es

*The $\boldsymbol{e}$-Xtra logo stands for "electronic extra" and indicates that four supplementary figures and three supplementary tables are published online.

() 2017 The American Phytopathological Society fixing root nodules. The establishment of efficient symbiosis is a complex process that requires continuous and precise signaling between the two partners (Downie 2010; Oldroyd et al. 2011). It is generally accepted that motility is not essential for nodulation or nitrogen fixation, although it is thought to be important in the initial stages of the symbiosis by directing bacteria to the proper infection sites. In agreement with this, different studies performed with nonflagellated, nonmotile, or nonchemotactic rhizobial strains showed that these bacteria were less competitive and less efficient in nodulation (Ames and Bergman 1981; Bernabéu-Roda et al. 2015; Caetano-Anollés et al. 1988, 1992; Mellor et al. 1987; Miller et al. 2007).

In the alfalfa endosymbiont Sinorhizobium meliloti, the genes required for flagellar motility (i.e., the flagellar regulon) are expressed in a transcriptional hierarchy. The master regulatory genes vis $N$ and vis $R$ of class IA and the regulatory rem gene belonging to class IB are at the top of the transcriptional cascade (Rotter et al. 2006; Sourjik et al. 2000). Expression of these class I genes is influenced by different regulators, including twocomponent regulatory systems (TCS), which participate in the coordinated control of motility and exopolysaccharide (EPS) synthesis in response to specific environmental conditions (Morris and González 2009; Yao et al. 2004).

In addition to the well-known swimming motility (flagelladependent movement of bacteria within liquid environments), $S$. meliloti can translocate over semisolid surfaces by employing different mechanisms, depending on the strain. Strains carrying a functional $\exp R$ gene exhibit an unusual quorum sensingcontrolled spreading (or surfing) which is mostly driven by physical or chemical effects created by the secreted EPS galactoglucan (EPS II) (Dilanji et al. 2014; Gao et al. 2012). ExpRdeficient strains such as the reference laboratory strain Rm1021 can also translocate over surfaces using at least two different types of motility. One type is flagellum-independent surface spreading or sliding, which is dependent on the siderophore rhizobactin 1021 (Nogales et al. 2010, 2012). The second type of surface translocation shown by $S$. meliloti Rm1021 is swarming, absolutely dependent on flagellar action and positively influenced by EPS (Nogales et al. 2012). Unlike $\mathrm{Rm} 1021, S$. meliloti strain GR4, which is an ExpR-deficient strain unable to produce the siderophore rhizobactin 1021, can translocate over semisolid surfaces by using a mechanism which is absolutely dependent on flagellar action (i.e., it only shows swarming motility) (Bernabéu-Roda et al. 2015). Swarming is a surface-associated multicellular behavior extensively studied in pathogenic bacteria due to its close connection with the 
virulence of these microorganisms (Verstraeten et al. 2008). In contrast, in $S$. meliloti, the genetic determinants controlling surface motility in general and swarming in particular as well as the role of this specialized type of translocation in the establishment of symbiosis are mostly unknown.

With the aim of identifying new molecular mechanisms governing swarming in S. meliloti, we isolated GR4-derived transposants with impaired surface motility. Here, we report on the characterization of one of these mutants (GRS577) in which the transposon interrupted the $n t r Y$ gene which codes for the histidine kinase of the NtrY/NtrX TCS. In other species, this system is mainly involved in the regulation of nitrogen metabolism. In Azorhizobium caulinodans, Azospirillum brasilense, Rhizobium tropici, Rhodobacter capsulatus, Ehrlichia chaffeensis, and Herbaspirillum seropedicae, the TCS is required to assimilate different nitrogen sources (Bonato et al. 2016; Cheng et al. 2014; Drepper et al. 2006; Ishida et al. 2002; Nogales et al. 2002; Pawlowski et al. 1991). In Azorhizobium caulinodans and $R$. capsulatus, the system is required for nitrogen fixation. In Brucella spp., NtrY/NtrX acts as a redox sensor that influences bacterial replication within eukaryotic cells (Carrica et al. 2012). In Brucella spp. as well as in Neisseria gonorrheae, the TCS regulates the expression of respiratory enzymes, including nitrogen respiration (denitrification) enzymes, and high-affinity cytochrome oxidases (Atack et al. 2013; Carrica et al. 2012, 2013). In addition to nitrogen metabolism, in the photosynthetic bacterium $R$. capsulatus, the NtrY/NtrX system also regulates the formation of photosynthetic complexes (Gregor et al. 2007). Interestingly, in S. meliloti strain Rm1021, the response regulator NtrX but not the histidine kinase NtrY was suggested to be involved in the regulation of succinoglycan (EPS I) production, motility, and symbiotic nitrogen fixation (Wang et al. 2013).

Results obtained during characterization of the $n t r Y:: \operatorname{Tn} 5 \mathrm{mu}-$ tant GRS577 described in this work support the notion that $n t r Y$ and $n t r X$ are genetically coupled in S. meliloti, and unveil a complex regulatory role for this TCS. Both genes participate in the control of flagella and EPS production, contribute to maintain cell membrane integrity, and are required for salt stress tolerance. In addition, under microaerobic conditions, the NtrY/NtrX system regulates nitrogen fixation and respiration genes. Importantly, our data indicate that the NtrY/NtrX system influences root colonization and competition for nodule occupancy on alfalfa plants but is not essential for symbiotic nitrogen fixation.

\section{RESULTS}

\section{Identification and characterization of GRS577, an $S$. meliloti GR4 ntrY::Tn5 mutant.}

To identify $S$. meliloti genes playing a role in swarming motility, strain GR4 was subject to Tn5 transposon mutagenesis. In total, 5.800 kanamycin-resistant transposants were tested for surface motility on semisolid minimal medium (MM). Fourteen mutants were identified that exhibited reduced surface motility relative to the wild-type (WT) strain (GR4 reduced swarming [GRS]) (Fig. 1A). Genetic characterization of these mutants revealed several genes potentially playing a role in the control of GR4 surface motility. Among them were genes involved in the turnover of second messengers, as well as uncharacterized genes coding for putative proteins involved in transport, central intermediary metabolism, or transcriptional regulation, or of unknown function. Interestingly, in 5 of the 14 mutants, the Tn5 insertion was interrupting the coding sequence of $n t r Y$ (smc01044) at three different positions (representative mutants GRS2175, GRS1980, and GRS577) (Fig. 1B). Here, we report on the characterization of GRS577.

Some of the free-living phenotypes shown by the GRS577 transposant were similar to those reported for an $n t r X$ insertion mutant derived from strain Rm1021 (Wang et al. 2013). Thus, GRS577 was defective in swimming motility (Fig. 2A) and produced fewer flagella $(0.6 \pm 0.2)$ than the parental strain GR4 (2.6 \pm 0.2$)$ (Fig. 2B). Additionally, GRS577 exhibits a different cell morphology compared with GR4. GRS577 cells were smaller and more spherical than WT cells (cell lengths of $1.66 \pm$ 0.06 and $1.94 \pm 0.06 \mu \mathrm{m}$, respectively) and they tended to form clusters (Fig. 2B). The altered morphology of GRS577 cells is suggestive of envelope defects. Sensitivity tests performed using sodium dodecyl sulfate (SDS) as a membrane stressor revealed increased sensitivity of the mutant to the detergent (Fig. 2C). Furthermore, GRS577 showed a mucoid appearance in $\mathrm{MM}$ and bright fluorescence on MM supplemented with the fluorescent stain Calcofluor (Fig. 2D), suggesting the overproduction of EPS I. This phenotype was nutrient dependent because it was not observed in rich tryptone yeast (TY) medium. Inactivation of the exoY gene, essential for EPS I biosynthesis, abolished the mucoid and fluorescent phenotypes associated with the ntrY::Tn5 mutation, demonstrating that succinoglycan overproduction was responsible for those traits (Fig. 2E).

Surprisingly, in contrast to the Rm1021 ntrX insertion mutant obtained by Wang et al. (2013), GRS577 was able to establish

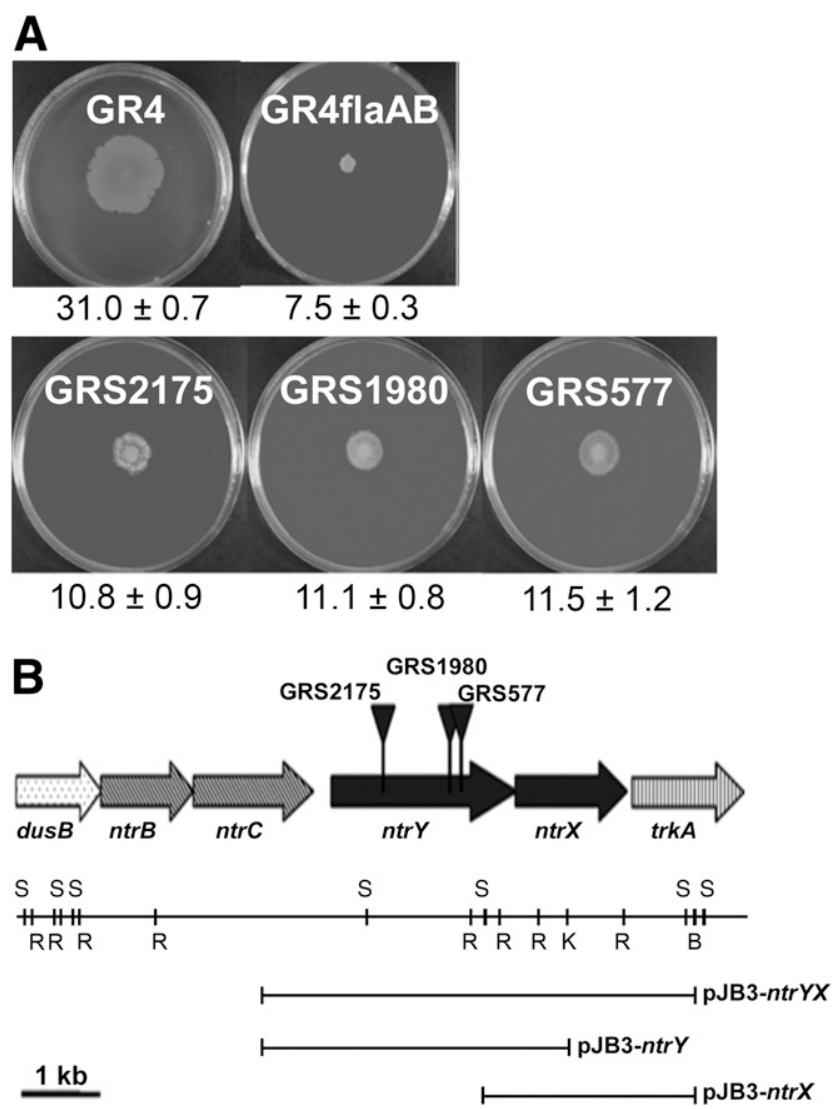

Fig. 1. Isolation and genetic characterization of Sinorhizobium meliloti GR4 transposon-induced mutants defective in surface motility. A, Surface motility exhibited by the wild-type strain GR4, the nonflagellated derivative strain GR4flaAB, and three transposon-induced mutants with reduced surface translocation. Pictures were taken $24 \mathrm{~h}$ after inoculation. Under each image, the mean and standard error of the migration zones (given in millimeters) obtained from at least 12 measurements taken from three independent experiments are indicated. B, Genomic context of the $S$. meliloti $n$ tr $Y$ gene. The Tn5 insertion of mutants GRS2175, GRS1980, and GRS577 is indicated with triangles. Segments under the physical map indicate the genomic DNA region present in plasmid constructs used in complementation experiments. Restriction sites shown are EcoRI (R), SphI (S), KpnI (K), and BamHI (B). 
efficient symbiosis with alfalfa plants (Supplementary Fig. S1). No significant differences were observed in shoot dry weight of plants inoculated with the mutant compared with that of plants inoculated with the parental strain GR4 (data not shown). Likewise, no differences could be detected in either the morphology or number of nodules developed by plant roots inoculated with GR4 or the GRS577 mutant.

\section{Both NtrY and NtrX participate in the control} of motility and EPS I production in S. meliloti GR4.

Experimental as well as in silico data indicate that the $n t r Y$ and $n t r X$ genes are cotranscribed in S. meliloti (Schlüter et al. 2013; Wang et al. 2013). In order to examine a possible effect on downstream gene expression caused by the Tn 5 insertion in GRS577, transcript levels of $n t r X$ were analyzed by reversetranscription quantitative polymerase chain reaction (RT-qPCR). Data revealed approximately 10-fold lower gene expression in the mutant than in the parental strain GR4 (Table 1). Likewise, transcript levels of $n t r Y$ sequences located downstream of the Tn5 insertion (ntrYDT) showed sixfold reduction in GRS577 compared with those in GR4. These results demonstrate that the transposon present in GRS577 was interrupting the transcription not only of the $n t r Y$ gene but also that of the downstream $n t r X$ gene. Transcription levels of $n t r Y$ sequences located upstream of the Tn5 insertion (ntrYUT) were also analyzed and found to be sevenfold higher in GRS577 than in GR4. These expression data suggest that the NtrY/NtrX system directly or indirectly autoregulates its own expression in $S$. meliloti.

In order to determine whether the different phenotypes shown by GRS577 were due to effects caused by the transposon in $n t r Y, n t r X$, or both genes, complementation experiments were carried out. Thus, different pJB3-derived plasmid constructs harboring either $n t r Y, n t r X$, or both genes (Fig. 1B) were transferred to GRS577 and the motility, mucoidy, fluorescence, and SDS sensitivity phenotypes of the corresponding transconjugants were examined for complementation. Only strain 577JYX, which harbors the pJB3-ntrYX construct, regained virtually all WT phenotypes (Fig. 3). To rule out the possibility that the absence of phenotypic complementation in GRS577 transconjugants harboring the pJB3-ntrX plasmid (strain $577 \mathrm{JX}$ ) could be caused by impaired $n t r X$ transcription, expression analysis were performed by RT-qPCR. Results showed that compared with the WT strain carrying the empty vector (GR4J), transcription of $n t r X$ was decreased in 577J (average relative expression of -3.6 with a standard error of \pm 0.9 ) but it was increased in 577JX (average relative expression of +33.7 with a standard error of \pm 5.3 ). Therefore, our complementation studies suggest that in $S$. meliloti GR4, both genes, ntrY and

Table 1. Relative expression of different genes in GRS577 compared with the wild-type strain GR4

\begin{tabular}{lc}
\hline Gene & Relative expression $^{\mathbf{a}}$ \\
\hline$n t r X$ & $-9.9 \pm 2.0$ \\
$n t r Y \mathrm{DT}^{\mathrm{b}}$ & $-6.0 \pm 1.2$ \\
$n t r Y \mathrm{UT}^{\mathrm{b}}$ & $7.0 \pm 0.8$ \\
vis $N$ & $-8.1 \pm 0.9$ \\
flaA & $-42.3 \pm 8.8$ \\
exoY & $14.7 \pm 1.8$ \\
\hline
\end{tabular}

${ }^{a}$ Fold changes in gene expression obtained from reverse-transcription quantitative polymerase chain reaction experiments. The smc03224 gene coding for $16 \mathrm{~S}$ ribosomal RNA was used as an internal control. Mean values and standard errors were calculated from at least two independent experiments with three technical replicates. The minus sign (-) indicates a decreased fold change in GRS577.

${ }^{\mathrm{b}} n t r Y \mathrm{DT}$ and $n t r Y \mathrm{UT}$ correspond to $n t r Y$ sequences located downstream and upstream of Tn5, respectively.
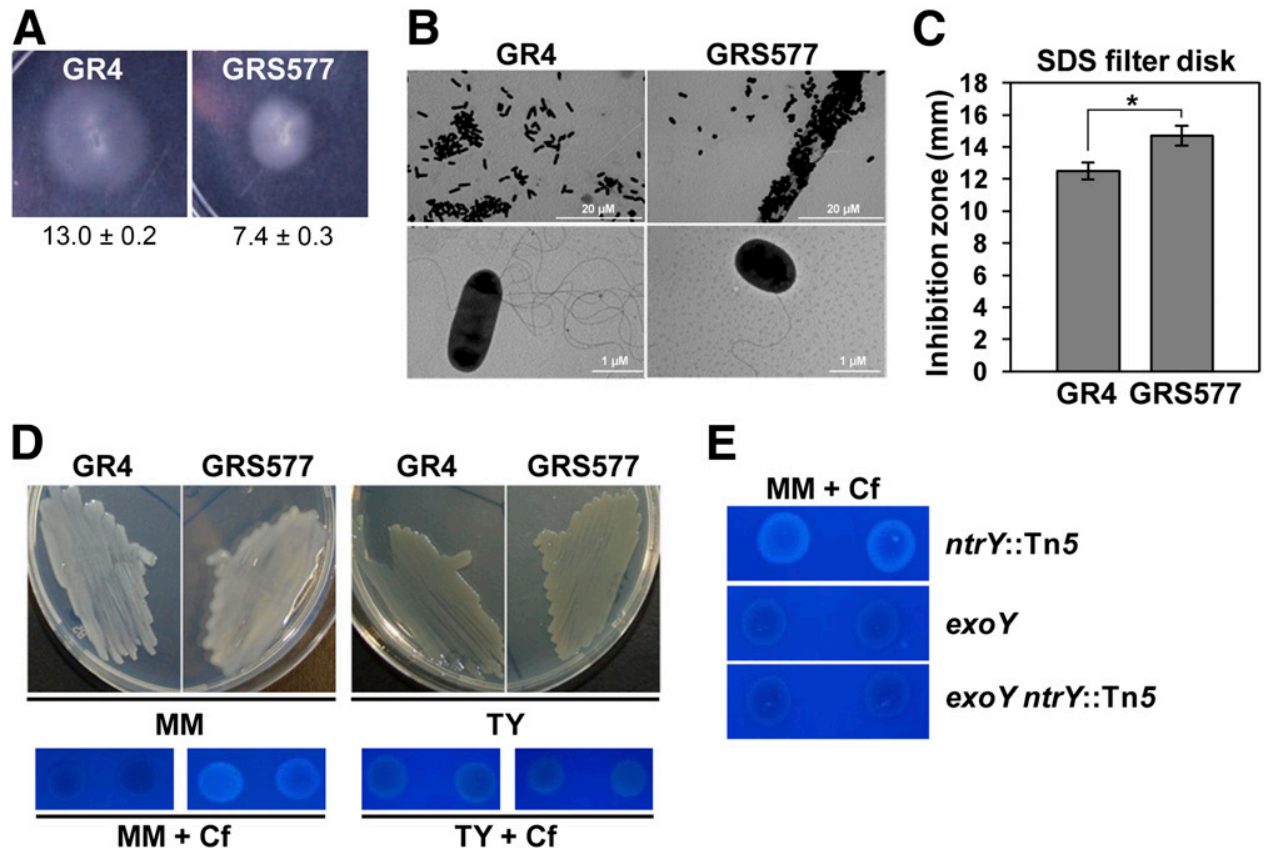

$\mathbf{E}$

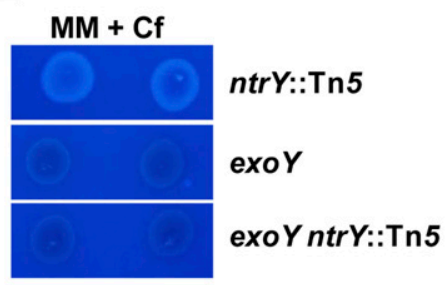

Fig. 2. A, Swimming motility assay of the wild-type strain GR4 and the ntrY::Tn5 mutant GRS577. Pictures were taken 72 h after inoculation. Under each image, the mean and standard error of the migration zones (given in millimeters) obtained from at least 12 measurements taken from three independent experiments are indicated. B, Transmission electron microscopy images showing the morphology and flagella production of wild-type and mutant cells grown on semisolid minimal medium (MM) for $24 \mathrm{~h}$. Scale bars are indicated. C, Sodium dodecyl sulfate (SDS) sensitivity test. Bars represent the mean of the growth inhibition (in millimeters) of GR4 and GRS577 caused by the presence of a filter disk soaked with 10\% SDS. Asterisk indicates significant differences according to an analysis of variance test $(P<0.05)$. D, Mucoid appearance (upper) and fluorescence (lower) exhibited by wild-type and mutant strains after growth on MM (left) or tryptone yeast (TY) (right) plates. To observe fluorescence after UV radiation, plates were supplemented with Calcofluor (Cf). E, Fluorescence shown by single $n t r Y:$ Tn5 (GRS577) and exoY (GR4Y) mutants and the double exoY ntrY::Tn5 (GR4YntrY) mutant grown on MM plates supplemented with Cf. 
$n t r X$, participate in the coordinated control of motility and succinoglycan production, as well as in resistance to membrane stressors.

In parallel to complementation experiments, and in order to determine the function of the NtrY/NtrX module in $S$. meliloti GR4, we tried to obtain the single $n t r Y$ deletion mutant as well as a double ntrYntrX deletion mutant. However, several attempts following standard allelic exchange techniques used routinely in $S$. meliloti were unsuccessful. According to Wang et al. (2013), NtrX but not NtrY is essential for the viability in strain Rm1021. To test the possibility that deletion of the $n t r Y$ gene could be lethal for strain GR4, the allelic exchange procedure was performed in strains carrying one of the different plasmids used in our complementation experiments. Deletion of the $n t r Y$ gene was achieved only in strains harboring the pJB3-ntrYX construct but not in those harboring the empty vector or the constructs carrying $n t r Y$ or $n t r X$ alone. Similar results were obtained when the same strategy for allelic replacement was used in the Rm1021 genetic background. It should be mentioned that, when allelic replacement was attempted in cells harboring pJB3-ntrY, some of the clones lacked the WT chromosomal allele (Supplementary Fig. S2, lane 5); however, the lack of signal corresponding to the pJB3borne $n \operatorname{tr} Y$ sequences, together with the presence of signals showing the expected size for the deleted version of $n \operatorname{tr} Y$ but too intense to be chromosomal borne, was suggestive of $n t r Y$ sequence rearrangements within cells. These data indicate that, under our experimental conditions, deletion of the complete $n t r Y$ gene sequence but not interruption by a transposon insertion is lethal in both GR4 and Rm1021 strains.

\section{GRS577 exhibits increased biofilm formation and high sensitivity to salt stress.}

It is known that $S$. meliloti EPS I overproducers exhibit greater biofilm formation on abiotic surfaces (Fujishige et al. 2006). In agreement with this, GRS577 showed an increased ability to develop biofilms on glass surfaces compared with the WT strain GR4 (Fig. 4A). Only the pJB3-ntrYX construct was able to suppress the increased biofilm formation ability shown by the GRS577 mutant, whereas plasmid constructs containing either only $n t r Y$ or only $n t r X$ did not. Abolition of EPS I synthesis in GR4YntrY (exoY and ntrY) reduced the amount of bacteria attached to glass surfaces to levels similar to those shown by the WT (Fig. 4A), indicating that EPS I overproduction was responsible for the increased biofilm formation ability exhibited by the mutant.

On the other hand, $n t r Y$ was identified as a gene involved in salt tolerance in the bean symbiont $R$. tropici CIAT899 (Nogales et al. 2002). Here, we show that the mutation present in GRS577 prevents the growth of $S$. meliloti in MM supplemented with $300 \mathrm{mM} \mathrm{NaCl}$ (Fig. 4B). Similar to the previously described phenotypes shown by GRS577, only introduction of the plasmidborne $n \operatorname{tr} Y X$ genes could rescue bacterial growth in the presence of high salt concentrations, assigning a role for the NtrY/NtrX regulatory system in salt tolerance in free-living bacteria.

\section{Motility defects in GRS577 hinder alfalfa root colonization and decrease competitive nodulation.}

As already mentioned, GRS577 was able to develop nitrogenfixing nodules in alfalfa plants. Because rhizobial motility has been associated with nodulation efficiency and competitiveness, we decided to investigate whether these symbiotic phenotypes were altered in the $n t r Y:: \operatorname{Tn} 5$ mutant, which was highly defective in flagellar synthesis and motility. In single inoculation experiments, GRS577 exhibited nodule formation efficiency similar to that of the WT strain GR4 (data not shown). However, in competition assays in which the mutant and WT strains were coinoculated in a 1:1 ratio, the mutant occupied approximately $50 \%$ fewer nodules than the WT (Fig. 5A). When inoculated in a 10:1 ratio (mutant/WT), the percentage of nodules occupied by the mutant was only $29 \%$, revealing its low ability to compete with GR4 for the nodulation of alfalfa plants (Fig. 5A). Complementation of the GRS577 mutant with pJB3-ntrYX restored its ability to compete with the WT (Fig. 5A). To gain further insights into the reasons for the low competitive ability shown by GRS577, the transposant's capacity to adhere to and colonize alfalfa roots was examined. No significant differences in initial attachment ( $2 \mathrm{~h}$ postinoculation) were detected between GR4 and GRS577 (Fig. 5B). However, with longer incubation periods, a clear difference in alfalfa rhizosphere colonization was
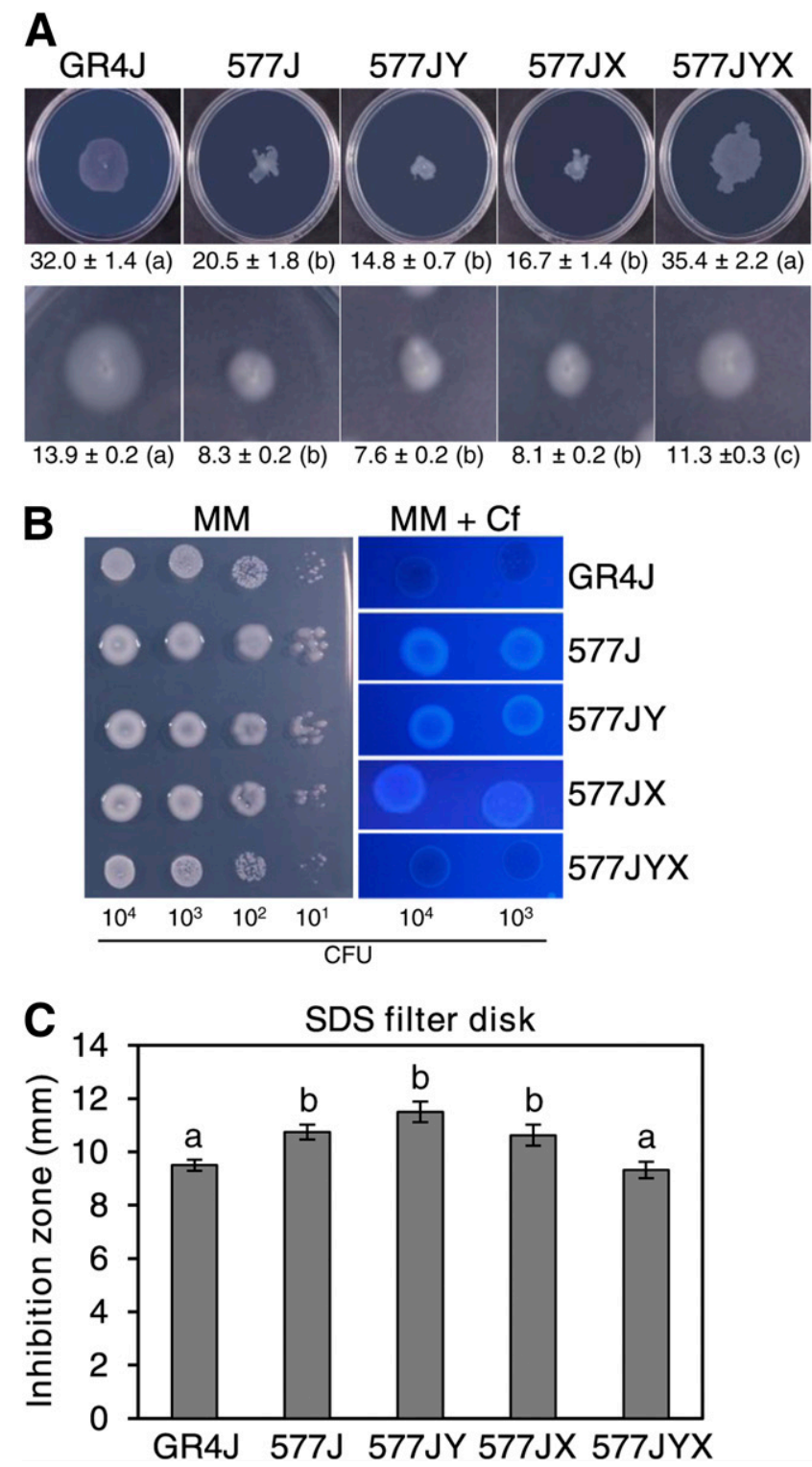

Fig. 3. Complementation of motility, exopolysaccharide I production, and sodium dodecyl sulfate (SDS) tolerance in GRS577. A, Surface motility (upper row) and swimming (lower row) assays. Under each image, the mean and standard error of the migration zones (given in millimeters) obtained from at least 18 measurements are indicated. Different letters indicate significant differences according to an analysis of variance test $(P<0.05)$. B, Mucoid appearance (left) and fluorescence (right) exhibited by different strains on minimal medium (MM) and MM supplemented with Calcofluor (Cf). The number of CFU that were plated in each lane is indicated. $\mathbf{C}$, Growth inhibition caused by the presence of filter disks soaked with $10 \%$ SDS. Different letters indicate significant differences $(t$ test, $P<0.05)$. 
observed, with the WT developing 10- to 100-fold more cells per gram of root tissue than GRS577 (Fig. 5B). This result indicates that the mutant was severely impaired in root colonization, which could give a clear advantage to the WT for nodule occupation in competition assays.

The motility impairment caused by reduced flagella production in GRS577 could be at least partially responsible for the poor root colonization shown by the mutant. We reasoned that, by increasing flagella production, nodule competitiveness could be rescued. Overexpression of the motility master regulator VisNR in GRS577 (strain 577TNR) increased the flagella number per cell and significantly improved the mutant's swimming and surface motilities, reaching levels similar to those shown by GR4 (Supplementary Fig. S3). Competition assays performed on alfalfa plants revealed that overexpression of VisNR was sufficient to recover the competitive nodulation ability of GRS577 to WT levels (Fig. 5A), indicating that the reduced competitiveness for nodule occupancy shown by the mutant was the result of an impaired bacterial motility.

\section{Transcriptome analysis of GRS577.}

In order to uncover genes that could potentially be regulated by the NtrY/NtrX system in S. meliloti GR4, the transcriptomes of GRS577 and WT GR4 cells grown in MM broth to mid exponential phase were compared. In total, 231 genes appeared as differentially expressed: $143(62 \%)$ were downregulated whereas $88(38 \%)$ were upregulated in GRS577 (Supplementary Table S1). Interestingly, an uneven distribution among the $S$. meliloti replicons was found for the up- and downregulated genes. Thus, $47.6 \%$ (68 of 143) of the genes showing lower expression in the mutant compared with the WT were located on megaplasmid pSymA, whereas $40.9 \%$ (36 of 88) of the upregulated genes belonged to megaplasmid $\mathrm{pSymB}$, percentages which are significantly higher than the 21 and $25 \%$, respectively, expected for an even distribution among the $S$. meliloti replicons. These results suggest that, under free-living conditions, the NtrY/NtrX system of $S$. meliloti promotes expression of genes located on pSymA whereas it represses genes located on $\mathrm{pSymB}$, an effect that could be either direct or indirect.

Among the downregulated genes in the GRS577 mutant, three major groups could be distinguished: the largest group comprised a total of 51 genes $(35 \%)$ that code for hypothetical proteins of unknown functions; a second group contained 33 genes $(23 \%)$ related to chemotaxis and flagellar motility and included class IA and IB transcriptional regulators visN and rem, respectively (Rotter et al. 2006; Sourjik et al. 2000); finally, a third group encompassed 28 pSymA-located genes (19\%) whose expression is induced in response to microaerobic conditions. Within the last group, the following genes were found: 9 genes involved in the denitrification pathway (nosR and the complete nosZDFYLX operon coding for the dissimilatory nitrous oxide reductase, as well as the first gene of the operons coding for a periplasmic nitrate reductase $[n a p E]$ and nitric oxide reductase $[$ norE] $) ; 2$ genes related to the denitrification process (azul and hemN) (Torres et al. 2014), 14 different fix genes, including copies 1 and 2 of the fixNOQP operon that encode the highaffinity $c b b_{3}$ oxidase required for respiration in the microoxic environment of the nodule (Bobik et al. 2006; Torres et al. 2013);

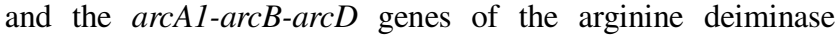
pathway, which is a widespread anaerobic route for arginine degradation (Bobik et al. 2006). It is also worth mentioning the downregulation of several genes responsible for tolerance to

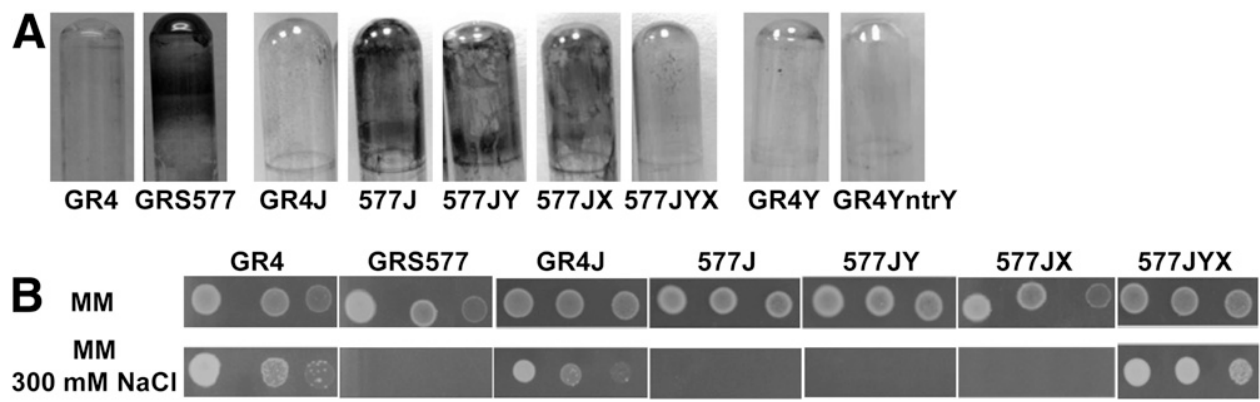

Fig. 4. A, Biofilm formation on glass tubes exhibited by Sinorhizobium meliloti strains in minimal medium (MM) broth. B, Ability of S. meliloti strains to grow in MM supplemented with $300 \mathrm{mM} \mathrm{NaCl}$. Drops containing approximately $10^{6}, 10^{5}$, and $10^{4} \mathrm{CFU}$ (from left to right) of each strain were applied onto the surface of $\mathrm{MM}$ (upper row) or MM supplemented with $300 \mathrm{mM} \mathrm{NaCl}$ (lower row) and incubated at $28^{\circ} \mathrm{C}$ for 3 days. A representative example of at least three experiments is shown.
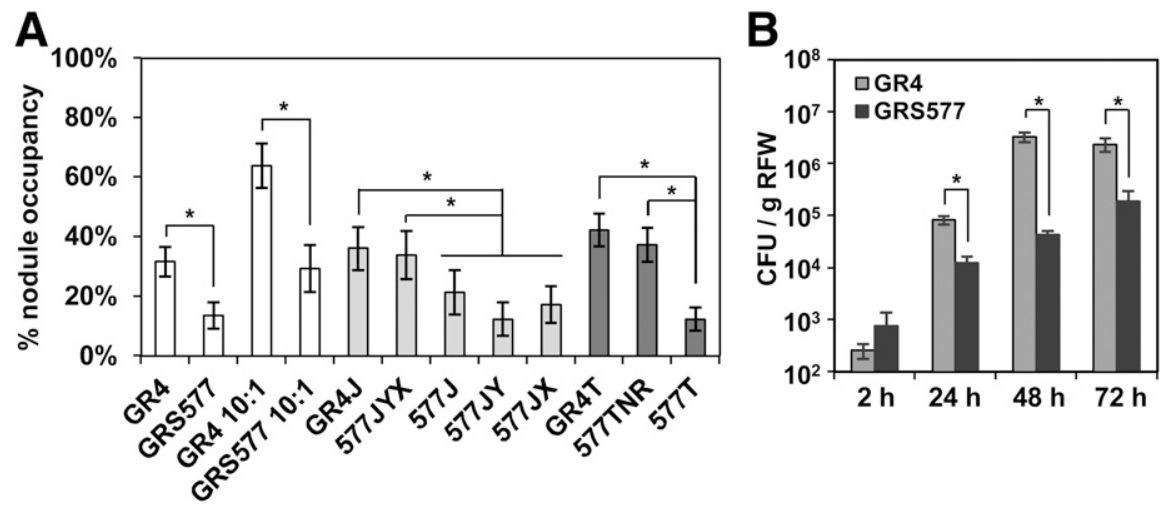

Fig. 5. A, Competitive nodulation assays. Data represent the percentage of nodules occupied by each rhizobial strain after coinoculation with the marked strain GR4 (pGUS3) in a mixture ratio of 1:1 (unmarked/pGUS3-marked) or 10:1 (indicated). B, Adsorption or colonization assays. Bars represent CFU counts recovered from alfalfa roots at different times after inoculation. Data are expressed per gram of root fresh weight (RFW). Error bars indicate the standard error from the mean obtained from three independent experiments. Asterisks indicate significant differences according to an analysis of variance test $(P<0.05)$. 
different stresses: $g s t 13, c l p B$, and heat- and cold-shock proteins, which could indicate that the mutant has a diminished capacity to cope with adverse environmental conditions. In addition, the presence of $n t r X$ within the group of downregulated genes in the GRS577 transcriptome confirmed our results obtained by RTqPCR that were mentioned above.

As observed for the group of downregulated genes, many of the upregulated genes $(34 \%)$ in the $n t r Y:: \operatorname{Tn} 5$ transposant code for hypothetical proteins of unknown function. In addition, the upregulation of 11 exo genes responsible for EPS I synthesis (exoY, exoQ, exoZ, exoH, exoN, exoO, exoP, exoU, exoV, and exoW) and depolymerization (exoK) (Janczarek 2011) was remarkable, a finding which is consistent with EPS I overproduction. The upregulation of several genes predicted to encode structural components of the cell envelope and cellwall-modifying enzymes (lys $M$, murF, mrcA2, ampC, rlpA, ropB1, sma2295, and smc04236) was also noteworthy. Confirming our previous data obtained by RT-qPCR (Table 1), the $n t r Y$ gene appeared among genes significantly overexpressed in GRS577 compared with the WT GR4 strain.

In addition to for the already mentioned $n t r Y$ and $n t r X$ genes, the microarray results of this study were further validated by confirming the differential expression of visN, flaA, and exoY by RT-qPCR (Table 1$)$.

\section{S. meliloti NtrY/NtrX regulates expression of denitrification and nifA genes.}

In S. meliloti, expression of denitrification genes (nap, nir, nor, and nos genes that encode the corresponding reductases for nitrate, nitrite, nitric oxide, and nitrous oxide, respectively) is induced under microaerobic conditions $\left(2 \% \mathrm{O}_{2}\right)$ and in the presence of nitrate (Becker et al. 2004; Torres et al. 2014). Although our transcriptomic study was performed with cells grown in wellaerated cultures and in the absence of nitrate, reduced expression of some denitrification genes (napE, norE, nosR, and nosZDFYLX) was detected in the GRS577 mutant compared with the WT. Interestingly, the NtrY/NtrX TCS of Brucella spp. has been involved in the induction of nitrate respiration enzymes under microaerobic conditions (Carrica et al. 2012). This prompted us to investigate whether $\mathrm{NtrY} / \mathrm{NtrX}$ could play a similar role in S. meliloti. Using transcriptional fusions to the lacZ reporter gene, we analyzed the expression of nirK (for nitrite reductase) and norB (for nitric oxide reductase) in S. meliloti GR4 and GRS577 cells after growth in liquid MM or MM supplemented with $\mathrm{KNO}_{3}$ under aerobic and microaerobic conditions $\left(2 \% \mathrm{O}_{2}\right)$. In addition, $\beta$-galactosidase activity of WT and mutant cells containing either plasmid pCHK57 (pnifA::lacZ) or pGD499 (pnpt::lacZ) were also measured as controls for microaerobic induction and constitutive gene expression, respectively. No significant differences between GR4 and GRS577 were detected concerning the expression of the constitutive npt promoter under any of the four experimental conditions tested (data not shown), thereby ruling out the existence of unspecific effects on gene expression. Under microaerobic conditions, the expression of the nifA gene increased eightfold in WT GR4 cells grown in MM, compared with the expression observed under aerobic conditions (Fig. 6A). In microaerobiosis, the presence of nitrate in culture medium reduced by approximately half the levels of nifA gene expression in GR4. This negative effect of nitrate on S. meliloti nifA expression under free-living microaerobic conditions has already been described previously (Fischer 1994; Noonan et al. 1992). In microaerobiosis and in the absence of nitrate, nifA expression levels in GRS577 were more than twofold lower than those observed for GR4 and, interestingly, they were not affected by the presence of nitrate (Fig. 6A). These results suggest that, under microaerobic conditions, the NtrY/NtrX system is required to achieve maximum expression of nifA in the absence of nitrate.

In the absence of nitrate, the expression of norB and nirK increased in both GR4 and GRS577 in response to microaerobiosis, reaching similar levels (Fig. 6B and C). The presence of nitrate under microaerobic conditions increased significantly the expression of norB (3-fold) and nirK (10-fold) in GR4 cells. Interestingly, although the presence of nitrate also induced expression of norB and nirK in GRS577 grown in microaerobiosis, expression levels of these genes were significantly reduced (1.7and 2.7-fold lower, respectively) compared with those observed in the WT GR4 grown under the same conditions (i.e., microaerobiosis and in the presence of nitrate). These results suggest that the NtrY/NtrX system is responsible for maximum expression of some denitrification enzymes in microaerobiosis when nitrate is present.

\section{DISCUSSION}

In this work, characterization of a surface motility-deficient mutant (GRS577) has extended the knowledge about the complex role played by the TCS NtrY/NtrX in the alfalfa symbiont. Our data indicate that, although not essential for establishing nitrogen-fixing symbiosis, both $n t r Y$ and $n t r X$ genes act together to regulate important traits in $S$. meliloti. Both genes are required for the expression of flagellar genes, control of EPS I production, cell envelope integrity, tolerance to salt stress, alfalfa root colonization, and competitive nodulation. In addition, the NtrY/NtrX system is required for optimal expression of nitrogen metabolism genes (nifA and denitrification genes) under microaerobic conditions.
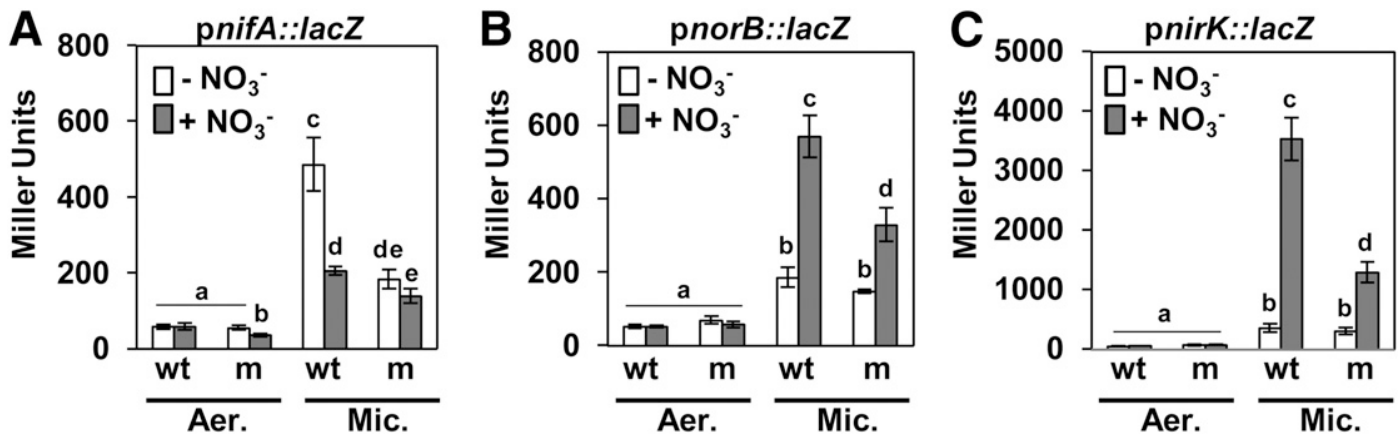

Fig. 6. Expression of transcriptional fusions to lacZ of the A, nifA; $\mathbf{B}$, norB; and $\mathbf{C}$, nirK genes in Sinorhizobium meliloti GR4 (wt) and its derivative mutant GRS577 (m). The activity of nifA, norB, and nirK promoter regions was measured in both strains after growth in liquid minimal medium (MM) (white bars) and

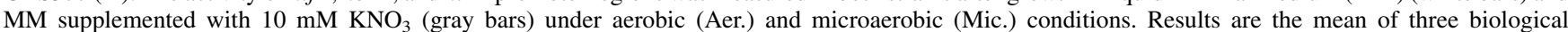
independent experiments with two technical replicates. Error bars represent standard errors. Different letters indicate significant differences according to an analysis of variance test $(P<0.05)$. 
Some of the conclusions reached in our study are significantly different from those reported recently concerning the role of NtrY/NtrX in S. meliloti strain Rm1021 (Wang et al. 2013). In Rm1021, NtrX (but not the histidine kinase NtrY) was suggested to be involved in motility, production of EPS I, and symbiotic nitrogen fixation. Deletion of the $n t r X$ gene was found to be lethal. Hence, results reported by Wang et al. (2013) were obtained using a plasmid insertion mutant which exhibited decreased $n t r X$ expression. In GRS577, the Tn5 insertion disrupts the coding sequence of the $n t r Y$ gene and also leads to reduced $n t r X$ expression levels. The decrease in $n t r X$ transcription detected in GRS577 is similar to that reported for the Rm1021 ntrX plasmid insertion mutant (10 and 14\% of the $n t r X$ transcriptional activity detected in GR4 and Rm1021, respectively). Wang and coworkers (2013) could restore the EPS I production and symbiotic phenotype of their $n t r X$ insertion mutant with a plasmid carrying the $n t r X$ gene under the control of the lac promoter. In agreement with a role for the $n t r Y$ gene, we could not complement GRS577 phenotypes using the plasmid construct pJB3-ntrX that allowed expression of the $n t r X$ gene from a lac promoter, as shown by RT-qPCR. Likewise, the construct pJB3-ntrY failed to complement the mutant. Only the pJB3-ntrYX construct, which expresses both $n t r Y$ and $n t r X$ genes, restored the different phenotypes exhibited by GRS577. These results indicate that both $n t r Y$ and $n t r X$ participate in the control of the different observed phenotypes.

According to Wang and coworkers (2013), $n t r Y$ is dispensable in $\mathrm{Rm} 1021$ and an $n t r Y$ deletion mutant behaves as the WT. However, under our experimental conditions, deletion of the chromosomal $n t r Y$ gene in either GR4 or Rm1021 strains by using standard allelic exchange techniques could only be achieved in the presence of the pJB3-ntrYX plasmid. Hybridization experiments revealed that, in the absence of pJB3ntrYX, some colonies resulting from allelic replacement selection contained unexpected rearrangements affecting $n t r Y$ sequences. These rearrangement phenomena could have escaped Wang and coworkers (2013) because their mutants were checked only by PCR. Curiously, attempts to obtain $n t r Y$ and $n t r X$ mutants in Azospirillum brasilense by double crossover events were also unsuccessful (Ishida et al. 2002). Although, in that study, potential candidates were obtained, hybridization analyses revealed that the cassette was inserted in a site different from the $n t r Y$ gene. Taking into account these and our own attempts to delete $n t r Y$, there is a possibility that the $n t r Y$ gene might still be present in the mutant obtained in Rm1021. Unfortunately, the mutations published by Wang et al. (2013) could not be tested under our experimental conditions. Our results indicate that complete deletion of $n t r Y$ compromises cell viability in $S$. meliloti.

GRS577 exhibits a pleiotropic phenotype. The decreased visNR expression and upregulation of exo genes are responsible for its impaired flagella-dependent motility and EPS I overproduction phenotypes. Moreover, compared with the parental strain, the mutant exhibited an increased ability to form biofilm on glass surfaces, a behavior that was demonstrated to be linked to EPS I overproduction. GRS577 shows increased salt sensitivity compared with the WT strain, a phenotype that was also described for an $n t r Y$ mutant of the bean symbiont Rhizobium tropici (Nogales et al. 2002). The altered morphology and increased sensitivity to membrane stressors shown by GRS577 suggest that the NtrY/NtrX system might be involved in maintaining cell envelope integrity. In agreement with this, the transcriptome profiling of the transposant revealed differential expression of several genes coding for predicted structural components of the cell envelope and cell-wall-modifying enzymes. Therefore, NtrY/NtrX adds to the increasing group of regulatory proteins identified in S. meliloti such as Emm, ExoRExoS/ChvI, or CbrA that coordinately control critical cell processes, including motility, EPS production, and free-living viability (Gibson et al. 2007; Morris and González 2009; Wang et al. 2010). Curiously, expression of the $c h v I$ gene was increased in GRS577 compared with the WT strain. However, a possible interaction between the NtrY/NtrX and the ExoR-ExoS/ChvI systems has not been investigated further in this study.

Importantly, we show that GRS577 establishes symbiosis with alfalfa as efficiently as the WT. The Rm1021 ntrX plasmid insertion mutant was shown to be defective in symbiotic nitrogen fixation in alfalfa plants, although nifA or nifH expression remained unaffected (Wang et al. 2013). The nature of the mutations harbored by the two mutant strains could explain their different symbiotic phenotypes. Very slight expression of $n t r X$ in GRS577, possibly from a promoter internal to Tn5, might be enough to support efficient symbiotic nitrogen fixation. Another possible explanation for the different symbiotic phenotypes exhibited by these two mutants, both of which are affected in the NtrY/NtrX system, is that the disruption of the normal functioning of just one mutant or the two components of the NtrY/NtrX system leads to different phenotypes. This effect, which could be the result of cross-talk between the $\mathrm{NtrY} / \mathrm{NtrX}$ and the upstream-lying NtrB/NtrC regulatory systems, has been described in Azorhizobium caulinodans (Pawlowski et al. 1991). In this bacterium, free-living $\mathrm{N}$ fixation is more severely affected by the removal of one activator protein (NtrC or $\mathrm{NtrX})$ than by the removal of the complete system (NtrY/NtrX). To solve the discrepancies, the Rm1021 ntrX plasmid insertion mutant needs further genetic examination.

Data in the literature about transcriptional regulation of $n t r Y$ and $n t r X$ genes is scarce. In A. caulinodans, $n t r Y X$ expression was shown to be dependent on $n t r C$ when cells were grown on nitrate (Pawlowski et al. 1991). In Rhodobacter capsulatus and Brucella abortus, transcription of $n t r Y$ starts within the $n t r C-n t r Y$ intergenic region but may also be transcribed from another promoter upstream of nifR3-ntrB-ntrC (Carrica et al. 2012; Drepper et al. 2006). To the best of our knowledge, there is no evidence indicating that $S$. meliloti $n t r Y X$ might be transcribed from a promoter upstream of $d u s B-n t r B-n t r C$. Transcript start site (TSS) mapping in $S$. meliloti did not reveal any TSS upstream of $d u s B$ $n t r B-n t r C$, although one TSS upstream of the start codon of $n t r C$ was mapped in addition to several TSS within the intergenic $n t r C$ $n t r Y$ region (Schlüter et al. 2013). Recently, in B. abortus, NtrX has been shown to repress transcription by binding sequences in the $n t r C$-ntrY intergenic region (Fernández et al. 2017). Our gene expression analyses suggest that the NtrY/NtrX system of $S$. meliloti is autoregulated. Interestingly, a DNA sequence resembling the $B$. abortus NtrX binding site could be identified upstream of $n t r Y$ overlapping two putative TSS (Supplementary Fig. S4). The possibility that $S$. meliloti $\mathrm{NtrX}$ can directly repress transcription of $n t r Y X$ requires further investigation.

Although no apparent deficiencies in symbiotic nitrogen fixation were associated with GRS577, this strain exhibited a reduced ability to compete with the WT strain for nodule occupancy. We have demonstrated that this behavior is the result of the motility impairment because overexpression of the flagellar VisNR master regulator was sufficient to complement bacterial competitiveness. Therefore, this study supports the notion that, although motility is not essential for nodulation and $\mathrm{N}$ fixation, it affects root colonization, a trait that can affect the establishment of symbiosis, especially when competing with other microorganisms.

The gene expression analyses performed in this work indicate that, in S. meliloti, the NtrY/NtrX system participates in fine-tuning nitrogen metabolism gene expression in response to specific environmental conditions. Under microaerobic conditions, when nitrate is absent, the system leads to maximum expression of the nifA gene, whereas maximum expression of 
denitrification genes requires the presence of nitrate. The negative effect of nitrate on S. meliloti nifA expression under free-living microaerobic conditions has been described previously (Fischer 1994; Noonan et al. 1992); however, this is the first time that the NtrY/NtrX system has been associated with this effect. Likewise, it was already known that the presence of nitrate and microaerobic conditions $\left(2 \% \mathrm{O}_{2}\right)$ can activate the expression of denitrification genes in $S$. meliloti (Torres et al. 2014). Our study links the NtrY/NtrX system to this process. Interestingly, the transcriptomic analyses of the GRS577 mutant also revealed the downregulation of several fixNOQP genes that encode the cytochrome $c$ oxidase $\left(c b b_{3}\right)$ with high affinity for $\mathrm{O}_{2}$ (Bobik et al. 2006; Torres et al. 2013). A role for the $\mathrm{NtrY} / \mathrm{NtrX}$ system in regulating the expression of nitrogen respiration enzymes and high-affinity cytochrome oxidase genes has already been described in the bacterial pathogen genera Brucella and Neisseria (Atack et al. 2013; Carrica et al. 2012, 2013) but not in the legume symbiont $S$. meliloti. Similar to these bacteria, the $S$. meliloti NtrY/NtrX system may contribute, together with other TCS such as ActSR (Fenner et al. 2004), to the adaptation to the microoxic conditions encountered during its symbiotic lifestyle within the root nodule.
In summary, this work has unveiled the role of the NtrY/NtrX system in controlling $S$. meliloti traits relevant for both freeliving and symbiotic states. Oxygen tension and the presence of nitrate modulate its regulatory role. Under free-living conditions, the system contributes to salt tolerance and to maintain cell envelope integrity. In addition, it is responsible for increased bacterial motility and repression of succinoglycan production and biofilm formation. This allows rhizobial cells to achieve efficient root colonization and optimal nodulation competitive ability. Although the NtrY/NtrX system is dispensable for symbiotic nitrogen fixation, it contributes to microaerobic energy metabolism by inducing high-affinity cytochrome oxidases and fine tuning nitrogen respiration enzymes as well as the machinery for $\mathrm{N}$-fixation.

\section{MATERIALS AND METHODS}

Bacterial strains, plasmids, and growth conditions.

Bacterial strains and plasmids used in this work and their relevant characteristics are listed in Table 2. Escherichia coli strains were grown in Luria-Bertani medium (Sambrook et al. 1989) at $37^{\circ} \mathrm{C} ; \mathrm{S}$. meliloti strains were grown at $30^{\circ} \mathrm{C}$ in either

Table 2. Bacterial strains and plasmids used in this study

\begin{tabular}{|c|c|c|}
\hline Strain or plasmid & Relevant characteristics $^{\mathbf{a}}$ & Reference or source \\
\hline \multicolumn{3}{|c|}{ Sinorhizobium meliloti } \\
\hline GR4 & Wild-type strain & Casadesús and Olivares 1979 \\
\hline GR4flaAB & GR4 flaAflaB::Hyg, $\mathrm{Hyg}^{\mathrm{r}}$ & Bernabéu-Roda et al. 2015 \\
\hline GRS577 & GR4 ntrY::Tn5, $\mathrm{Km}^{\mathrm{r}}$ & This work \\
\hline GRS2175 & GR4 ntrY::Tn5, $\mathrm{Km}^{\mathrm{r}}$ & This work \\
\hline GRS1980 & GR4 ntrY::Tn5, $\mathrm{Km}^{\mathrm{r}}$ & This work \\
\hline GR4Y & GR4 $\triangle$ exoY & This work \\
\hline GR4YntrY & GR4Y containing the $n t r Y:: \operatorname{Tn} 5$ mutation from GRS2175, $\mathrm{Km}^{\mathrm{r}}$ & This work \\
\hline GR4J & GR4 containing plasmid pJB3Tc19, $\mathrm{Tc}^{\mathrm{r}}$ & This work \\
\hline $577 \mathrm{~J}$ & GRS577 containing plasmid pJB3Tc19, $\mathrm{Km}^{\mathrm{r}}, \mathrm{Tc}^{\mathrm{r}}$ & This work \\
\hline $577 \mathrm{JY}$ & GRS577 containing plasmid pJB3-ntrY, $\mathrm{Km}^{\mathrm{r}}, \mathrm{Tc}^{\mathrm{r}}$ & This work \\
\hline $577 \mathrm{JX}$ & GRS577 containing plasmid pJB3-ntrX, $\mathrm{Km}^{\mathrm{r}}, \mathrm{Tc}^{\mathrm{r}}$ & This work \\
\hline $577 J Y X$ & GRS577 containing plasmid pJB3-ntrYX, $\mathrm{Km}^{\mathrm{r}}, \mathrm{Tc}^{\mathrm{r}}$ & This work \\
\hline GR4T & GR4 containing plasmid pTE3, $\mathrm{Tc}^{\mathrm{r}}$ & This work \\
\hline $577 \mathrm{~T}$ & GRS577 containing plasmid pTE3, $\mathrm{Km}^{\mathrm{r}}, \mathrm{Tc}^{\mathrm{r}}$ & This work \\
\hline 577TNR & GRS577 containing plasmid pTE3-visNR, $\mathrm{Km}^{\mathrm{r}}, \mathrm{Tc}^{\mathrm{r}}$ & This work \\
\hline \multicolumn{3}{|l|}{ Escherichia coli } \\
\hline DH5 $\alpha$ & supE44, DlacU169, f80, lacZDM, 5hsdR171, recA1, endA1, gyrA96, thi-1, relA1 & Bethesda Research Lab \\
\hline S17-1 & thi, pro, recA, hsdR, hsdM, RP4-2-Tc::Mu-Km::Tn7 & Simon et al. 1983 \\
\hline \multicolumn{3}{|l|}{ Plasmids } \\
\hline pUC18 & Cloning vector, $\mathrm{Ap}^{\mathrm{r}}$ & Yanisch-Perron et al. 1985 \\
\hline pCR-XL-TOPO & Cloning vector, $\mathrm{Km}^{\mathrm{r}}$ & Invitrogen \\
\hline pSUP202 & $\mathrm{pBR} 325:: \mathrm{mob}, \mathrm{Ap}^{\mathrm{r}}, \mathrm{Tc}^{\mathrm{r}}, \mathrm{Cm}^{\mathrm{r}}$ & Simon et al. 1983 \\
\hline pSUP2021 & pSUP202 derivative with $\mathrm{Tn} 5, \mathrm{Ap}^{\mathrm{r}}, \mathrm{Tc}^{\mathrm{r}}, \mathrm{Cm}^{\mathrm{r}}, \mathrm{Km}^{\mathrm{r}}$ & Simon et al. 1983 \\
\hline pK18mobsacB & Suicide plasmid, $\mathrm{Km}^{\mathrm{r}}$ & Schäfer et al. 1994 \\
\hline pK18- $\Delta$ exoY & pK18mobsacB carrying the deleted version of the exoY locus, $\mathrm{Km}^{\mathrm{r}}$ & Nogales et al. 2012 \\
\hline pK18- $\Delta$ ntrY & pK18mobsacB carrying the deleted version of the $n t r Y$ locus, $\mathrm{Km}^{\mathrm{r}}$ & This work \\
\hline pJB3Tc19 & IncP cloning vector, $\mathrm{Tc}^{\mathrm{r}}, \mathrm{Ap}^{\mathrm{r}}$ & Blatny et al. 1997 \\
\hline pTE3 & IncP broad-host-range expression vector with $\operatorname{trp}$ promoter, $\mathrm{Tc}^{\mathrm{r}}$ & Egelhoff and Long 1985 \\
\hline pMP220 & IncP transcriptional fusion vector, $\mathrm{Tc}^{\mathrm{r}}$ & Spaink et al. 1987 \\
\hline pGD499 & $n p t:: l a c Z$ transcriptional fusion vector, $\mathrm{Tc}^{\mathrm{r}}$ & Ditta et al. 1985 \\
\hline pCHK57 & nifA::lac $Z$ transcriptional fusion vector, $\mathrm{Tc}^{\mathrm{r}}$ & Ditta et al. 1987 \\
\hline pTOPO-ntrYX & pCR-XL-TOPO carrying S. meliloti ntrYX genes, $\mathrm{Km}^{\mathrm{r}}$ & This work \\
\hline pTOPO-visNR & pCR-XL-TOPO carrying S. meliloti visNR genes, $\mathrm{Km}^{\mathrm{r}}$ & This work \\
\hline pUC-E2175 & $\begin{array}{l}\text { pUC18 carrying the EcoRI fragment that contains the } n t r Y:: \operatorname{Tn} 5 \text { mutation of } \\
\text { GRS2175; } \mathrm{Ap}^{\mathrm{r}}, \mathrm{Km}^{\mathrm{r}}\end{array}$ & This work \\
\hline pSUP202-E2175 & pSUP202 carrying the EcoRI insert of pUC-E2175, $\mathrm{Ap}^{\mathrm{r}}, \mathrm{Tc}^{\mathrm{r}}$ & This work \\
\hline pJB3-ntrYX & pJB3Tc19 with $S$. meliloti ntr $Y X$ genes cloned downstream from the lacZ promoter, $\mathrm{Tc}^{\mathrm{r}}$ & This work \\
\hline pJB3-ntrY & pJB3-ntrYX derivative in which part of the $n t r X$ coding sequence has been deleted, $\mathrm{Tc}^{\mathrm{r}}$ & This work \\
\hline pJB3-ntrX & pJB3Tc19 with the $n t r X$ gene cloned downstream from the lacZ promoter, $\mathrm{Tc}^{\mathrm{r}}$ & This work \\
\hline pTE3-visNR & $\begin{array}{l}\text { pTE3 derivative harboring S. meliloti visNR genes downstream from the trp } \\
\text { promoter, } \mathrm{Tc}^{\mathrm{r}}\end{array}$ & This work \\
\hline pMP-Smnor & pMP220 derivative carrying a norECB::lac $Z$ transcriptional fusion, $\mathrm{Tc}^{\mathrm{r}}$ & This work \\
\hline pMP-Smnir & pMP220 derivative carrying a nirK: :lacZ transcriptional fusion, $\mathrm{Tc}^{\mathrm{r}}$ & This work \\
\hline pGUS3 & Plasmid which contains an $n f e D:: g u s A$ fusion, $\mathrm{Km}^{\mathrm{r}}$ & García-Rodríguez and Toro 2000 \\
\hline
\end{tabular}

\footnotetext{
${ }^{\mathrm{a}} \mathrm{Hyg}^{\mathrm{r}}, \mathrm{Km}^{\mathrm{r}} \mathrm{Ap}^{\mathrm{r}}, \mathrm{Tc}^{\mathrm{r}}$, and $\mathrm{Cm}^{\mathrm{r}}$, indicate hygromycin, kanamycin, ampicillin, tetracycline, and chloramphenicol resistance, respectively.
} 
complex TY medium (Beringer 1974), Bromfield medium (BM) $(0.04 \%$ tryptone, $0.01 \%$ yeast extract, and $0.01 \%$ $\mathrm{CaCl}_{2} \cdot 2 \mathrm{H}_{2} \mathrm{O}$ ), or MM (Robertsen et al. 1981). To detect the overproduction of EPS I, Calcofluor-White M2R (fluorescent brightener 28; Sigma-Aldrich) was added to TY or MM plates at a final concentration of $0.02 \%$. Salt tolerance was determined by spot inoculation of 10-fold serial dilutions of each strain on solid MM plates supplemented with $300 \mathrm{mM} \mathrm{NaCl}$. When required, antibiotics were added at final concentrations of ampicillin at $200 \mu \mathrm{g} \mathrm{ml}^{-1}$, kanamycin at $50 \mu \mathrm{g} \mathrm{ml}^{-1}$, and tetraclycline at $10 \mu \mathrm{g} \mathrm{ml}^{-1}$ for E. coli; and kanamycin at $200 \mu \mathrm{g} \mathrm{m}{ }^{-1}$, tetracycline at $10 \mu \mathrm{g} \mathrm{ml}^{-1}$, and hygromycin at $75 \mu \mathrm{g} \mathrm{ml}^{-1}$ for $S$. meliloti. To improve reproducibility, all liquid cultures of $S$. meliloti were initiated from glycerol stocks.

\section{Isolation of $S$. meliloti mutants with altered surface motility.}

Transposon Tn 5 mutagenesis of $S$. meliloti GR4 was carried out following the procedure described by Simon et al. (1983), using strain S17-1 carrying pSUP2021 as donor. After selection on MM plates supplemented with kanamycin, individual transposants were grown in liquid TY in a Bioscreen $\mathrm{C}$ apparatus to late logarithmic phase (optical density at $600 \mathrm{~nm}$ $\left[\mathrm{OD}_{600}\right]=0.9$ to 1$)$. Each culture $(2 \mu \mathrm{l})$ was applied onto the surface of MM plates containing $0.6 \%$ purified agar, allowed to dry for $10 \mathrm{~min}$, and incubated overnight at $30^{\circ} \mathrm{C}$. Those clones showing decreased surface motility relative to WT strain GR4 in three independent experiments were selected for further characterization. The point of insertion for Tn 5 mutants was determined by arbitrary PCR, as described previously (MillerWilliams et al. 2006).

\section{Construction of plasmids and $S$. meliloti strains.}

Primers used to obtain the different plasmid constructs and $S$. meliloti mutant strains are listed in Supplementary Table S2.

For the construction of pJB3-ntrYX, a 5,070-bp fragment containing $S$. meliloti GR4 $n t r Y$ and $n t r X$ genes as well as $484 \mathrm{bp}$ of $n t r Y$ upstream sequences was amplified from genomic DNA using primers delntrYX.1 and delntrYX.4. The resulting PCR product was first cloned into pCR-XL-TOPO and sequenced, then subcloned as a BamHI fragment into pJB3Tc19, keeping $n t r Y n t r X$ transcription in the same orientation as lacZ. To obtain pJB3-ntrY, the pJB3-ntrYX plasmid was digested with KpnI to remove a fragment of 1,620 bp containing the $3^{\prime}$ half of the $n t r X$ coding sequence. For the construction of pJB3-ntrX, a 2,590-bp SphI fragment from pTOPO-ntrYX comprising the coding sequence of $n t r X$ was isolated and subcloned into pJB3Tc19 in the same orientation as lacZ.

The pTE-visNR plasmid was constructed by cloning a 1,812-bp PCR fragment containing visN and visR into BamHI-digested pTE3 vector, in the same orientation as the trp promoter. The fragment comprising the visN and visR genes and flanked by $B g l \mathrm{II}$ linkers was PCR amplified using Rm1021 genomic DNA and primers visNR-U and visNR-R, cloned into pCR-XL-TOPO, and sequenced before subcloning into pTE3.

To obtain plasmids pMP-Smnor and pMP-Smnir harboring norECB::lacZ and nirK::lacZ transcriptional fusions, respectively, DNA fragments of 2,056 and 852 bp flanked by EcoRI linkers were PCR amplified using GR4 genomic DNA and primer pairs pnor.F/pnor.R and pnirK.F/pnirK.R. Each fragment was cloned into EcoRI-digested pMP220, and clones harboring the insert in the correct orientation relative to the lac Z gene were selected.

For the construction of the EPS I-defective strain GR4Y, plasmid pK18- $\Delta$ exoY (Nogales et al. 2012) was introduced into GR4 via conjugation with $E$. coli strain S17-1 and allele replacement was selected as described previously (Schäfer et al.
1994). To obtain the GR4YntrY strain, plasmid pSUP202E2175 carrying the genomic EcoRI fragment harboring the $n t r Y:: T n 5$ mutation present in GRS2175 was introduced into GR4Y by conjugation, and allele exchange was selected. The Tn5 insertions isolated in this study could not be transduced into GR4 due to unavailability of adequate phages for this $S$. meliloti strain.

The deleted version of $n t r Y$ was generated by overlap extension PCR using primers delntrY-1 to delntrY-4. The resulting fusion product, in which a deletion of $2.1 \mathrm{~kb}$ was created, was cloned into pCR-XL-TOPO and sequenced. Then, the insert was subcloned into vector pK18mobsacB as an EcoRI fragment, yielding plasmid $\mathrm{pK} 18-\Delta \mathrm{ntrY}$. This construction was introduced into the desired $S$. meliloti strains by conjugation and the allelic exchange was selected in the absence or presence of different pJB3-derived constructs.

All mutants obtained in this work were checked by Southern hybridization with specific probes.

\section{Motility assays.}

Except for the initial screening of transposants (see above), surface motility of $S$. meliloti strains was assayed essentially as described by Bernabéu-Roda et al. (2015). Briefly, S. meliloti cells grown in TY broth to late logarithmic phase $\left(\mathrm{OD}_{600}=1\right.$ to 1.2) were pelleted, washed twice in MM, and resuspended in 0.1 volume of the latter medium. Aliquots of this bacterial suspension $\left(2 \mu \mathrm{l}\right.$, approximately $2 \times 10^{7}$ cells) were dispensed and allowed to dry for 10 min onto the surface of plates containing $20 \mathrm{ml}$ of semisolid MM containing $0.6 \%$ Noble Agar Difco (BD), which were previously air dried at room temperature for $15 \mathrm{~min}$. Surface migration was determined as the average length of the two sides of a rectangle able to exactly frame each colony. Swimming motility was examined on plates prepared with BM containing $0.3 \%$ Bacto agar and inoculated with $3-\mu l$ droplets of rhizobial cultures grown in $\mathrm{TY}\left(\mathrm{OD}_{600}=\right.$ 1). For swimming tests, the migration zone was determined as the colony diameter in millimeters after $72 \mathrm{~h}$ of incubation.

\section{Transmission electron microscopy (TEM).}

Cells for TEM observations were obtained from the edge of swarming colonies. Carbon-coated Formvar grids were placed for $5 \mathrm{~min}$ on top of a drop of water previously applied to the colony border. The grids were then washed twice in water for $1 \mathrm{~min}$ and stained with $2 \%$ (wt/vol) uranyl acetate for $3 \mathrm{~min}$. Grids were allowed to air dry for at least $1 \mathrm{~h}$ and visualized using a JEOL JEM-1011 transmission electron microscope with a $100-\mathrm{kV}$ beam at the Microscopy Service of the Estación Experimental del Zaidín, Granada, Spain. Images were captured using an Orius Gatan charge-coupled-device camera. Data presented for cell length and the number of flagella per cell are mean values and standard errors of at least 30 measurements.

\section{SDS-sensitivity assays.}

$S$. meliloti strains were grown in TY broth up to an $\mathrm{OD}_{600}$ of 0.9 to 1 , then diluted to an $\mathrm{OD}_{600}$ of 0.2 in the same medium. The diluted cultures $(100 \mu \mathrm{l})$ were inoculated in $3 \mathrm{ml}$ of softagar TY $(0.6 \%)$ and poured onto Petri plates containing $20 \mathrm{ml}$ of the same solid medium. The overlay was allowed to solidify for $30 \mathrm{~min}$. After that, a 5-mm paper disk was soaked with $5 \mu \mathrm{l}$ of $10 \%$ SDS (wt/vol) and placed on the center of the plate. The diameter of growth inhibition was recorded after $48 \mathrm{~h}$ of incubation at $30^{\circ} \mathrm{C}$.

\section{Biofilm assays.}

Stationary-phase cultures of $S$. meliloti strains grown in TY broth were washed with liquid MM and diluted 1:10 in glass 
tubes containing $4 \mathrm{ml}$ of fresh MM broth. Tubes were incubated for $48 \mathrm{~h}$ at $30^{\circ} \mathrm{C}$ under shaking conditions and for one additional week under static conditions at room temperature. After the incubation period, liquid from the tubes was removed by aspiration and bacterial biofilm stained with $8 \mathrm{ml}$ of $0.1 \%$ crystal violet for $20 \mathrm{~min}$. Tubes were washed carefully three times with deionized water to eliminate the excess of crystal violet, and air dried before pictures were taken.

\section{RNA isolation and synthesis of cDNA.}

Total RNA was isolated from $10 \mathrm{ml}$ of MM broth cultures at midlogarithmic phase $\left(\mathrm{OD}_{600}=0.5\right)$. Harvested cells were washed with $0.1 \%$ sarkosyl and pellets were immediately frozen with liquid nitrogen and stored at $-80^{\circ} \mathrm{C}$ until RNA extraction. RNA was isolated using the Qiagen RNeasy RNA purification kit (Qiagen) following the manufacturer's instructions. Residual DNA was removed with RNase-free Dnase I Set (Roche). The quality of the RNA was checked by $1.4 \%$ agarose gel electrophoresis.

For microarray hybridizations, RNA samples were concentrated using Microcon-30 filters (Millipore), after which Cy3and Cy5-labeled cDNA were prepared as previously described (Nogales et al. 2010) from 7.5 $\mu \mathrm{g}$ of total RNA.

\section{Microarray hybridization, image acquisition, and data analysis.}

Sm14kOLIGO microarrays, which are based on the Rm1021 genome, were purchased from A. Becker (University of Bielefeld, Bielefeld, Germany). Hybridizations with cDNA from GR4 strains were performed as described previously (Nogales et al. 2010). The genomes of GR4 and Rm1021 are similar but the latter lacks the two accessory plasmids pRmeGR4a and pRmeGR4b (Martínez-Abarca et al. 2013). For image acquisition, a GenePix 4100A Scanner (Axon Instruments, Inc.) was used. Quantifications of mean signal intensities for each spot were determined using the GenePix Pro software (Axon Instruments, Inc.). Normalization and $t$ statistics were carried out using the EMMA 2.8 microarray data analysis software developed at the Bioinformatics Resource Facility Center for Biotechnology, Bielefeld University. Data were collected and analyzed from three independent biological replicates. Genes were regarded as differentially expressed if they showed a $P$ value $\leq 0.05$, average signal to noise value $\geq 7$, and $\log _{2}$ experiment/control ratio value $\geq$ 1 or $\leq-1$. Detailed protocols and raw data resulting from the microarray experiments have been deposited in the ArrayExpress database with the accession number E-MTAB-2511.

\section{RT-qPCR.}

RNA $(1 \mu \mathrm{g})$ was reverse transcribed using Superscript II RT (Invitrogen) and random hexamers (Roche) as primers. Realtime qPCR was performed on an iCycler iQ5 (Bio-Rad). Each $25-\mu \mathrm{l}$ reaction contained $1 \mu \mathrm{l}$ of either a 0 or $1: 10000$ dilution (for amplification of the 16S ribosomal RNA [rRNA] gene) of cDNA, $200 \mathrm{nM}$ each primer, and the iQ SyBrGreen Supermix (Bio-Rad). Control PCR of the RNA samples not treated with RT were also performed to confirm the absence of contaminating genomic DNA. Samples were initially denatured by heating at $95^{\circ} \mathrm{C}$ for $3 \mathrm{~min}$ followed by a 35-cycle amplification and quantification program $\left(95^{\circ} \mathrm{C}\right.$ for $30 \mathrm{~s}, 55^{\circ} \mathrm{C}$ for $45 \mathrm{~s}$, and $72^{\circ} \mathrm{C}$ for $45 \mathrm{~s}$ ). A melting curve was conducted to ensure amplification of a single product. The oligonucleotide sequences for qPCR are listed in Supplementary Table S3. The efficiency for each primer pair $(\mathrm{E})$ was determined by running 10 -fold serial dilutions (four dilution series) of GR4 genomic DNA as template and generating a standard curve by plotting the log of the dilution factor against the cycle threshold $\left(\mathrm{C}_{\mathrm{T}}\right)$ value during the amplification of each dilution. Amplification efficiency was calculated using the formula $\mathrm{E}=\left[10^{(1 / \alpha)}-1\right] \times 100$, where $\alpha$ is the slope of the standard curve. The relative expression of each gene was normalized to that of 16S rRNA and the analysis of the results was performed using the comparative critical threshold $\left(\Delta \Delta \mathrm{C}_{\mathrm{T}}\right)$ method (Pfaffl 2001).

\section{$\beta$-Galactosidase activity measurement.}

Stationary-phase cultures of $S$. meliloti containing lacZ fusion plasmids grown in TY broth were washed and diluted 100fold in $3 \mathrm{ml}$ of either MM broth or MM broth supplemented with $10 \mathrm{mM} \mathrm{KNO}_{3}$. Tetracycline was added to all liquid cultures to ensure the maintenance of plasmid fusions. Cultures were incubated overnight at $30^{\circ} \mathrm{C}$ up to an $\mathrm{OD}_{600}=0.4$ to 0.6 . After the incubation period, 100- $\mu$ l aliquots of these cultures were used to determine $\beta$-galactosidase activity of cells grown under aerobic conditions. The remaining bacterial culture was subjected to microoxic conditions by gassing with $2 \%$ oxygen and $98 \%$ argon and incubated for $6 \mathrm{~h}$ at $30^{\circ} \mathrm{C}$. $\beta$-Galactosidase activity was determined by the SDS-chloroform method described by Miller (1972).

\section{Plant assays.}

Alfalfa (Medicago sativa L. 'Aragon') plants were surface sterilized, germinated, and grown in hydroponic cultures under axenic conditions in glass tubes containing nitrogen-free nutrient solution, as previously described (Olivares et al. 1980). To test the degree of infectivity of each strain, 12-day-old plants (a total of 24 replicates, one plant per tube) were inoculated with $1 \mathrm{ml}$ of a rhizobial suspension containing $5 \times 10^{6}$ cells. Prior to this inoculation, bacteria were grown to the exponential phase $\left(\mathrm{OD}_{600}=0.5\right.$ to 0.6$)$ in TY broth and diluted 100 -fold in sterile water. After inoculation, the number of nodules per plant was daily recorded. To examine competitive ability, 12 individual plants grown as described for the infectivity test were inoculated with $1 \mathrm{ml}$ of a bacterial suspension prepared as already described, containing mixtures at ratios $1: 1$ or 10:1 of the $S$. meliloti strain to be tested and GR4 marked with the pGUS3 plasmid. Roots were collected 15 days after inoculation, briefly washed with water, and incubated overnight in the dark at $37^{\circ} \mathrm{C}$ in $1 \mathrm{mM}$ 5-bromo-4-chloro-3-indolyl-b-D-glucuronide (Apollo Scientific) in $50 \mathrm{mM}$ sodium phosphate buffer ( $\mathrm{pH} 7.5)$ with $1 \%$ SDS. Those nodules occupied by the strain harboring the pGUS3 plasmid stained blue; thus, nodule occupancy could be determined by counting blue and white nodules produced by each bacterial mixture in at least two independent experiments.

For adhesion and colonization assays, 1-week-old alfalfa plants (five plants in each tube) were inoculated with $1 \mathrm{ml}$ of a rhizobial suspension as described above. At different times, 15 roots from each treatment were collected and washed three times with sterile distilled water, and pools of five plants were introduced to an Eppendorf tube containing $1 \mathrm{ml}$ of sterile TrisEDTA buffer (10 mM Tris-HCl [pH 7.5] and $1 \mathrm{mM}$ EDTA). Tubes were sonicated $(2 \times$ for $1 \mathrm{~min}$ each) in a Selecta Ultrasons sonicator bath. Released rhizobia were quantified by plate counting of serial dilutions of the supernatant, and means of the three determinations were plotted as the number of CFU per gram of root fresh weight.

To evaluate symbiotic nitrogen fixation of the different $S$. meliloti strains, alfalfa plants were grown in Leonard jars. One month after inoculation, the appearance and dry weight of the aerial part of plants as well as the aspect and number of nodules were compared.

\section{ACKNOWLEDGMENTS}

This work was supported by grants BIO2010-18005 and BIO201342801-P from the Spanish Ministry MICINN/MINECO, CVI-03541 from 
the Junta de Andalucía (Spain), and FEDER funds. J. Nogales was supported by a contract from MICINN (Spain) and N. Calatrava-Morales was supported by an FPU fellowship from MECD (Spain). TEM observations were carried out at the Microscopy service of the Estación Experimental del Zaidín (CSIC), Granada, Spain. We thank M. J. Delgado and M. J. Torres for their help in establishing microoxic conditions, and P. van Dillewijn and L. Bernabéu for critical reading of the manuscript.

\section{LITERATURE CITED}

Ames, P., and Bergman, K. 1981. Competitive advantage provided by bacterial motility in the formation of nodules by Rhizobium meliloti. J. Bacteriol. 148:728-908.

Atack, J. M., Srikhanta, Y. N., Djoko, K. Y., Welch, J. P., Hasri, N. H. M., Steichen, C. T., Vanden Hoven, R. N., Grimmond, S. M., Othman, D. S. M. P., Kappler, U., Apicella, M. A., Jennings, M. P., Edwards, J. L., and McEwan, A. G. 2013. Characterization of an $n t r X$ mutant of Neisseria gonorrhoeae reveals a response regulator that controls expression of respiratory enzymes in oxidase-positive proteobacteria. J. Bacteriol. 195:2632-2641.

Becker, A., Bergès, H., Krol, E., Bruand, C., Rüberg, S., Capela, D., Lauber, E., Meilhoc, E., Ampe, F., de Bruijn, F. J., Fourment, J., Francez-Charlot, A., Kahn, D., Küster, H., Liebe, C., Pühler, A., Weidner, S., and Batut, J. 2004. Global changes in gene expression in Sinorhizobium meliloti 1021 under microoxic and symbiotic conditions. Mol. Plant-Microbe Interact. 17:292-303.

Beringer, J. E. 1974. R factor transfer in Rhizobium leguminosarum. J. Gen. Microbiol. 84:188-198

Bernabéu-Roda, L., Calatrava-Morales, N., Cuéllar, V., and Soto, M. J. 2015. Characterization of surface motility in Sinorhizobium meliloti: Regulation and role in symbiosis. Symbiosis 67:79-90.

Blatny, J. M., Brautaset, T., Winther-Larsen, H. C., Haugan, K., and Valla S. 1997. Construction and use of a versatile set of broad-host-range cloning and expression vectors based on the RK2 replicon. Appl. Environ. Microbiol. 63:370-379.

Bobik, C., Meilhoc, E., and Batut, J. 2006. FixJ: A major regulator of the oxygen limitation response and late symbiotic functions of Sinorhizobium meliloti. J. Bacteriol. 188:4890-4902.

Bonato, P., Alves, L. R., Osaki, J. H., Rigo, L. U., Pedrosa, F. O., Souza, E. M., Zhang, N., Schumacher, J., Buck, M., Wassem, R., and Chubatsu, L. S. 2016. The NtrY-NtrX two-component system is involved in controlling nitrate assimilation in Herbaspirillum seropedicae strain SmR1. FEBS J. 283:3919-3930.

Caetano-Anollés, G., Wall, L. G., De Micheli, A. T., Macchi, E. M., Bauer, W. D., and Favelukes, G. 1988. Role of motility and chemotaxis in efficiency of nodulation by Rhizobium meliloti. Plant Physiol. 86: 1228-1235.

Caetano-Anollés, G., Wrobel-Boerner, E., and Bauer, W. D. 1992. Growth and movement of spot inoculated Rhizobium meliloti on the root surface of alfalfa. Plant Physiol. 98:1181-1189.

Carrica, Mdel. C., Fernandez, I., Martí, M. A., Paris, G., and Goldbaum, F. A. 2012. The NtrY/X two-component system of Brucella spp. acts as a redox sensor and regulates the expression of nitrogen respiration enzymes. Mol. Microbiol. 85:39-50.

Carrica, Mdel. C., Fernandez, I., Sieira, R., Paris, G., and Goldbaum, F. A. 2013. The two-component systems PrrBA and NtrYX co-ordinately regulate the adaptation of Brucella abortus to an oxygen-limited environment. Mol. Microbiol. 88:222-233.

Casadesús, J., and Olivares, J. 1979. Rough and fine linkage mapping of the Rhizobium meliloti chromosome. Mol. Gen. Genet. 174:203-209.

Cheng, Z., Lin, M., and Rikihisa, Y. 2014. Ehrlichia chaffeensis proliferation begins with NtrY/NtrX and PutA/GlnA upregulation and CtrA degradation induced by proline and glutamine uptake. mBio 5: e02141-14.

Dilanji, G. E., Teplitski, M., and Hagen, S. J. 2014. Entropy-driven motility of Sinorhizobium meliloti on a semi-solid surface. Proc. R. Soc. B 281 : 20132575.

Ditta, G., Schmidhauser, T., Yakobson, E., Lu, P., Liang, X. W., Finlay, D. R., Guiney, D., and Helinski, D. R. 1985. Plasmids related to the broad host range vector, pRK290, useful for gene cloning and for monitoring gene expression. Plasmid 13:149-153.

Ditta, G., Virts, E., Palomares, A., and Kim, C. H. 1987. The nifA gene of Rhizobium meliloti is oxygen regulated. J. Bacteriol. 169:3217-3223.

Downie, J. A. 2010. The roles of extracellular proteins, polysaccharides and signals in the interactions of rhizobia with legume roots. FEMS Microbiol. Rev. 34:150-170.

Drepper, T., Wiethaus, J., Giaourakis, D., Gross, S., Schubert, B., Vogt, M., Wiencek, Y., McEwan, A. G., and Masepohl, B. 2006. Cross-talk towards the response regulator NtrC controlling nitrogen metabolism in Rhodobacter capsulatus. FEMS Microbiol. Lett. 258:250-256.

Egelhoff, T. T., and Long, S. R. 1985. Rhizobium meliloti nodulation genes: Identification of nodDABC gene products, purification of nodA protein, and expression of nodA in Rhizobium meliloti. J. Bacteriol. 164:591-599.

Fenner, B. J., Tiwari, R. P., Reeve, W. G., Dilworth, M. J., and Glenn, A. R. 2004. Sinorhizobium medicae genes whose regulation involves the ActS and/or ActR signal transduction proteins. FEMS Microbiol. Lett. 236:21-31.

Fernández, I., Cornaciu, I., Carrica, M. D., Uchikawa, E., Hoffmann, G., Sieira, R., Márquez, J. A., and Goldbaum, F. A. 2017. Three-dimensional structure of full-length NtrX, an unusual member of the NtrC family of response regulators. J. Mol. Biol. 429:1192-1212.

Fischer, H. M. 1994. Genetic regulation of nitrogen fixation in rhizobia. Microbiol. Rev. 58:352-386.

Fujishige, N. A., Kapadia, N. N., De Hoff, P. L., and Hirsch, A. M. 2006. Investigations of Rhizobium biofilm formation. FEMS Microbiol. Ecol. 56:195-206.

Gao, M., Coggin, A., Yagnik, K., and Teplitski, M. 2012. Role of specific quorum-sensing signals in the regulation of exopolysaccharide II production within Sinorhizobium meliloti spreading colonies. PLoS One 7:e42611.

García-Rodríguez, F. M., and Toro, N. 2000. Sinorhizobium meliloti nfe (nodulation formation efficiency) genes exhibit temporal and spatial expression patterns similar to those of genes involved in symbiotic nitrogen fixation. Mol. Plant-Microbe Interact. 13:583-591.

Gibson, K. E., Barnett, M. J., Toman, C. J., Long, S. R., and Walker, G. C. 2007. The symbiosis regulator CbrA modulates a complex regulatory network affecting the flagellar apparatus and cell envelope proteins J. Bacteriol. 189:3591-3602.

Gregor, J., Zeller, T., Balzer, A., Haberzettl, K., and Klug, G. 2007. Bacterial regulatory networks include direct contact of response regulator proteins: Interaction of RegA and NtrX in Rhodobacter capsulatus. J. Mol. Microbiol. Biotechnol. 13:126-139.

Ishida, M. L., Assumpção, M. C., Machado, H. B., Benelli, E. M., Souza, E. M., and Pedrosa, F. O. 2002. Identification and characterization of the two-component NtrY/NtrX regulatory system in Azospirillum brasilense. Braz. J. Med. Biol. Res. 35:651-661.

Janczarek, M. 2011. Environmental signals and regulatory pathways that influence exopolysaccharide production in rhizobia. Int. J. Mol. Sci. 12: 7898-7933.

Martínez-Abarca, F., Martínez-Rodríguez, L., López-Contreras, J. A., Jiménez-Zurdo, J. I., and Toro, N. 2013. Complete genome sequence of the alfalfa symbiont Sinorhizobium/Ensifer meliloti strain GR4. Genome Announc. 1:e00174-12.

Mellor, H. Y., Glenn, A. R., Arwas, R., and Dilworth, M. J. 1987. Symbiotic and competitive properties of motility mutants of Rhizobium trifolii TA1. Arch. Microbiol. 148:34-39.

Miller, J. 1972. Experiments in Molecular Genetics. Cold Spring Harbor Laboratory Press, Cold Spring Harbor, NY.

Miller, L. D., Yost, C. K., Hynes, M. F., and Alexandre, G. 2007. The major chemotaxis gene cluster of Rhizobium leguminosarum bv. viciae is essential for competitive nodulation. Mol. Microbiol. 63:348-362.

Miller-Williams, M., Loewen, P. C., and Oresnik, I. J. 2006. Isolation of salt-sensitive mutants of Sinorhizobium meliloti strain Rm1021. Microbiology 152:2049-2059.

Morris, J., and González, J. E. 2009. The novel genes emmABC are associated with exopolysaccharide production, motility, stress adaptation, and symbiosis in Sinorhizobium meliloti. J. Bacteriol. 191:5890-5900.

Nogales, J., Bernabéu-Roda, L., Cuéllar, V., and Soto, M. J. 2012. ExpR is not required for swarming but promotes sliding in Sinorhizobium meliloti. J. Bacteriol. 194:2027-2035.

Nogales, J., Campos, R., BenAbdelkhalek, H., Olivares, J., Lluch, C., and Sanjuan, J. 2002. Rhizobium tropici genes involved in free-living salt tolerance are required for the establishment of efficient nitrogen-fixing symbiosis with Phaseolus vulgaris. Mol. Plant-Microbe Interact. 15: 225-232.

Nogales, J., Domínguez-Ferreras, A., Amaya-Gómez, C. V., van Dillewijn, P., Cuéllar, V., Sanjuán, J., Olivares, J., and Soto, M. J. 2010 Transcriptome profiling of a Sinorhizobium meliloti fadD mutant reveals the role of rhizobactin 1021 biosynthesis and regulation genes in the control of swarming. BMC Genomics 11:157.

Noonan, B., Motherway, M., and O'Gara, F. 1992. Ammonia regulation of the Rhizobium meliloti nitrogenase structural and regulatory genes under free-living conditions: Involvement of the fixL gene product? Mol. Gen Genet. 234:423-428.

Oldroyd, G. E., Murray, J. D., Poole, P. S., and Downie, J. A. 2011. The rules of engagement in the legume-rhizobial symbiosis. Annu. Rev. Genet. 45:119-144. 
Olivares, J., Casadesús, J., and Bedmar, E. J. 1980. Method for testing degree of infectivity of Rhizobium meliloti strains. Appl. Environ. Microbiol. 39:967-970.

Pawlowski, K., Klosse, U., and de Bruijn, F. J. 1991. Characterization of a novel Azorhizobium caulinodans ORS571 two-component regulatory system, NtrY/NtrX, involved in nitrogen fixation and metabolism. Mol. Gen. Genet. 231:124-138.

Pfaffl, M. W. 2001. A new mathematical model for relative quantification in real-time RT-PCR. Nucleic Acids Res. 29:e45.

Robertsen, B. K., Åman, P., Darvill, A. G., McNeil, M., and Alberstein, P. 1981. The structure of acidic extracellular polysaccharides secreted by Rhizobium leguminosarum and Rhizobium trifolii. Plant Physiol. 67: 389-400.

Rotter, C., Mühlbacher, S., Salamon, D., Schmitt, R., and Scharf, B. 2006. Rem, a new transcriptional activator of motility and chemotaxis in Sinorhizobium meliloti. J. Bacteriol. 188:6932-6942.

Sambrook, J., Fritsch, E. F., and Maniatis, T. 1989. Molecular Cloning: A Laboratory Manual. Cold Spring Harbor Laboratory Press, Cold Spring Harbor, NY.

Schäfer, A., Tauch, A., Jäger, W., Kalinowski, J., Thierbach, G., and Pühler, A. 1994. Small mobilizable multi-purpose cloning vectors derived from the Escherichia coli plasmids pK18 and pK19: Selection of defined deletions in the chromosome of Corynebacterium glutamicum. Gene 145:69-73.

Schlüter, J. P., Reinkensmeier, J., Barnett, M. J., Lang, C., Krol, E., Giegerich, R., Long, S. R., and Becker, A. 2013. Global mapping of transcription start sites and promoter motifs in the symbiotic $\alpha$-proteobacterium Sinorhizobium meliloti 1021. BMC Genomics 14:156.

Simon, R., Priefer, U., and Pühler, A. 1983. A broad host range mobilization system for in vivo genetic engineering: Transposon mutagenesis in Gram-negative bacteria. Nat. Biotechnol. 1:784-791.

Sourjik, V., Muschler, P., Scharf, B., and Schmitt, R. 2000. VisN and VisR are global regulators of chemotaxis, flagellar, and motility genes in Sinorhizobium (Rhizobium) meliloti. J. Bacteriol. 182:782-788.
Spaink, H. P., Okker, R. J., Wijffelman, C. A., Pees, E., and Lugtenberg, B. J. 1987. Promoters in the nodulation region of the Rhizobium leguminosarum Sym plasmid pRL1JI. Plant Mol. Biol. 9:27-39.

Torres, M. J., Hidalgo-García, A., Bedmar, E. J., and Delgado, M. J. 2013. Functional analysis of the copy 1 of the fixNOQP operon of Ensifer meliloti under free-living micro-oxic and symbiotic conditions. J. Appl. Microbiol. 114:1772-1781.

Torres, M. J., Rubia, M. I., de la Peña, T. C., Pueyo, J. J., Bedmar, E. J., and Delgado, M. J. 2014. Genetic basis for denitrification in Ensifer meliloti. BMC Microbiol. 14:142-151.

Verstraeten, N., Braeken, K., Debkumari, B., Fauvart, M., Fransaer, J., Vermant, J., and Michiels, J. 2008. Living on a surface: Swarming and biofilm formation. Trends Microbiol. 16:496-506.

Wang, C., Kemp, J., Da Fonseca, I. O., Equi, R. C., Sheng, X., Charles, T. C., and Sobral, B. W. 2010. Sinorhizobium meliloti 1021 loss-offunction deletion mutation in $c h v I$ and its phenotypic characteristics. Mol. Plant-Microbe Interact. 23:153-160.

Wang, D., Xue, H., Wang, Y., Yin, R., Xie, F., and Luo, L. 2013. The Sinorhizobium meliloti ntrX gene is involved in succinoglycan production, motility, and symbiotic nodulation on alfalfa. Appl. Environ. Microbiol. 79:7150-7159.

Yanisch-Perron, C., Vieira, J., and Messing, J. 1985. Improved M13 phage cloning vectors and host strains: Nucleotide sequences of the M13mp18 and pUC19 vectors. Gene 33:103-119.

Yao, S. Y., Luo, L., Har, K. J., Becker, A., Rüberg, S., Yu, G. Q., Zhu, J. B., and Cheng, H. P. 2004. Sinorhizobium meliloti ExoR and ExoS proteins regulate both succinoglycan and flagellum production. J. Bacteriol. 186: 6042-6049.

\section{AUTHOR-RECOMMENDED INTERNET RESOURCE}

University of Bielefeld, Bielefeld, Germany: http://emma.cebitec.uni-bielefeld.de 